\title{
General Analytic Solution of the Telegrapher's Equations and the Resulting Consequences for Electrically Short Transmission Lines
}

\author{
Steffen Kühn \\ Independent Scientist, Berlin, Germany \\ Email: steffen.kuehn@quantino-theory.de
}

How to cite this paper: Kühn, S. (2020) General Analytic Solution of the Telegrapher's Equations and the Resulting Consequences for Electrically Short Transmission Lines. Journal of Electromagnetic Analysis and Applications, 12, 71-87.

https://doi.org/10.4236/jemaa.2020.126007

Received: May 10, 2020

Accepted: June 12, 2020

Published: June 15, 2020

Copyright $\odot 2020$ by author(s) and Scientific Research Publishing Inc. This work is licensed under the Creative Commons Attribution International License (CC BY 4.0).

http://creativecommons.org/licenses/by/4.0/

\begin{abstract}
Based on classical circuit theory, this article develops a general analytic solution of the telegrapher's equations, in which the length of the cable is explicitly contained as a freely adjustable parameter. For this reason, the solution is also applicable to electrically short cables. Such a model has become indispensable because a few months ago, it was experimentally shown that voltage fluctuations in ordinary but electrically short copper lines move at signal velocities that are significantly higher than the speed of light in a vacuum. This finding contradicts the statements of the special theory of relativity but not, as is shown here, the fundamental principles of electrical engineering. Based on the general transfer function of a transmission line, the article shows mathematically that an unterminated, electrically short cable has the characteristics of an ideal delay element, meaning that an input signal appears at the output with a slight delay but remains otherwise unchanged. Even for conventional cables, the time constants can be so small that the corresponding signal velocities can significantly exceed the speed of light in a vacuum. The article also analyses the technical means with which this effect can be conveyed to very long cables.
\end{abstract}

\section{Keywords}

Telegrapher's Equations, Transmission Line Theory, Special Theory of Relativity, Electrically Short Transmission Lines, FTL Communication

\section{Introduction}

Some months ago, the author of this article discovered experimentally by coincidence that low-frequency voltage signals propagate in electrically short and 
unterminated transmission lines at phase and group velocities far faster than the speed of light in a vacuum. This is interesting, but not unique, because there are other known effects for which phase and group velocities are faster than light, as demonstrated in [1] [2] [3]. However, these effects cannot be exploited to transmit messages at speeds faster than the speed of light in a vacuum. This is not the case for the newly discovered effect, because it is not based on nonlinearities in the phase velocity as a function of the frequency. Instead, and this is remarkable, it can be shown mathematically that both the phase velocity and the group velocity are frequency independent and equal in a wide frequency band. If a signal is transmitted in this frequency band, it always retains its shape even though it moves much faster than light.

To better understand this unexpected effect, the author initially performed numerical simulations with the software SPICE. It turned out that the simulation results matched the measurement results very well. From this, it could be concluded that this is an effect of classical physics. It was therefore obvious that the effect should be explainable with classical network analysis. For this reason, the author carried out a corresponding mathematical analysis. The results are summarized in this article.

The mathematical analysis begins with a study of a transmission line model consisting of discrete components (lumped circuit). Subsequently, the discretization of the network is eliminated by limit calculation. As it turns out, this step leads to a previously unknown and very general solution of the telegrapher's equations, in which the length of the line is explicitly contained as a parameter in the solution.

With this solution, it is possible to analyze how the properties of a transmission line change smoothly from electrically short to electrically long. Up to now, the propagation velocities of signals in electrically short lines have usually been neglected as irrelevant in engineering. For long lines, such as power grids, however, the known and in their validity limited solutions of the telegrapher's equations are used.

The general solution of the telegrapher's equations derived in this article now makes it possible to study transmission lines that are neither electrically long nor electrically short. It becomes clear that electrically short and unterminated lines behave like delay elements. This means that the signal shape does not change when the signal passes the transmission line, and thus signal velocity, front velocity, phase velocity and group velocity are identical. Remarkably, this velocity can be far faster than the speed of light in a vacuum. According to the special theory of relativity, signal velocities higher than the speed of light in a vacuum are not possible, since this would lead to contradictions inside of the framework of special relativity.

However, for an engineer, the general solution of the telegrapher's equations derived in this article is a valuable tool for calculating phase, group and signal velocities for a given line length and given line constants. Further- 
more, it provides clear information on how to design spatially long transmission lines that allow data to be transmitted with much lower latency than it would be possible with optical fibers. That this seems to contradict the special theory of relativity is not discussed in this article and should be clarified elsewhere.

\section{Solution of the Telegrapher's Equations}

This article analyses the telegrapher's equations [4] under conditions that are usually ignored in the scientific literature [5]. To create a mathematically sound basis, a general solution is developed in this work. Although the method used for this purpose seems to be absent in the current technical literature it nonetheless proves to be very effective and compact. The starting point is the lumped circuit, as shown in Figure 1.

$Z_{L}$ is the series impedance per segment, and $Z_{Q}$, the corresponding shunt impedance. The parameter $n$ determines the number of segments. The terminating impedance $Z_{T}$, which is only present once at the end of the transmission line, has a special significance. In the case of an unterminated transmission line or a transmission line connected to a measuring device or circuit with a very high input resistance, the terminating impedance $Z_{T}$ is real and assumed to be infinite.

The total impedance $Z(n)$ of the circuit in Figure 1 can be expressed recursively by

$$
Z(n)=Z_{L}+\frac{1}{\frac{1}{Z_{Q}}+\frac{1}{Z(n-1)}}
$$

with

$$
Z(1)=Z_{L}+\frac{1}{\frac{1}{Z_{Q}}+\frac{1}{Z_{T}}} .
$$

The impedance over which the voltage $u_{1}$ drops has the value $Z(n)-Z_{L}$. However, the input voltage $u_{0}=U_{0} \mathrm{e}^{j \omega t}$ drops across the total impedance $Z(n)$ of the circuit. For this reason, the relation

$$
U_{1}=\frac{Z(n)-Z_{L}}{Z(n)} \cdot U_{0}
$$

applies to the complex amplitude or phasor $U_{1}$ of the voltage $u_{1}=U_{1} \mathrm{e}^{j \omega t}$. For

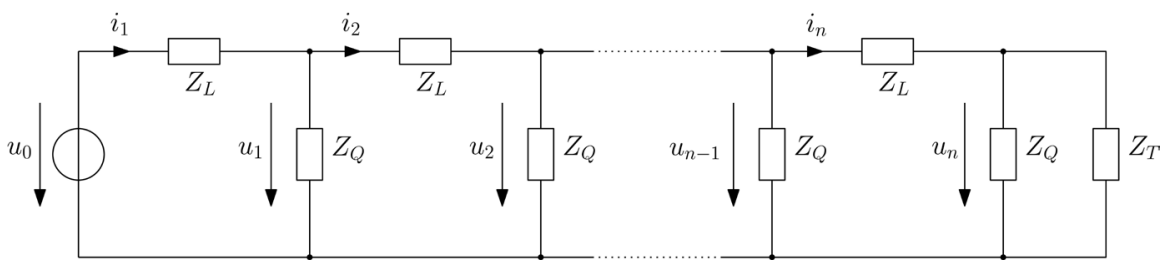

Figure 1. Lumped circuit model of the transmission line before the calculation of the limit. 
the complex amplitude $U_{2}$, the equation

$$
U_{2}=\frac{Z(n-1)-Z_{L}}{Z(n-1)} \cdot U_{1} .
$$

holds true. Thus, in general,

$$
U_{k}=G(k, n) \cdot U_{0}
$$

with

$$
G(k, n):=\prod_{i=1}^{k} \frac{Z(n-i+1)-Z_{L}}{Z(n-i+1)} .
$$

By inserting different values of $n$, it can be verified that for $G(k, n)$ the relation

$$
G(k, n)=\frac{2^{k}\left(\left(\alpha_{-}^{n-k}+\alpha_{+}^{n-k}\right) \beta_{1}-\left(\alpha_{-}^{n-k}-\alpha_{+}^{n-k}\right) \beta_{2}\right)}{\left(\alpha_{-}^{n}+\alpha_{+}^{n}\right) \beta_{1}-\left(\alpha_{-}^{n}-\alpha_{+}^{n}\right) \beta_{2}}
$$

is valid. For readability reasons, the abbreviations

$$
\begin{gathered}
\beta_{1}:=\sqrt{4+\frac{Z_{L}}{Z_{Q}}} Z_{T}, \\
\beta_{2}:=\sqrt{\frac{Z_{L}}{Z_{Q}}}\left(2 Z_{Q}+Z_{T}\right)
\end{gathered}
$$

and

$$
\alpha_{ \pm}:=\left(2 \pm \sqrt{\frac{Z_{L}}{Z_{Q}}} \sqrt{4+\frac{Z_{L}}{Z_{Q}}}+\frac{Z_{L}}{Z_{Q}}\right)
$$

are introduced. A formal proof is not given here, because at the end of this section, it is shown that the final result of the calculation is a valid solution of the telegrapher's equations.

To obtain a most realistic model, it is desirable that the number of segments is infinite for a given finite total length $l$ of the transmission line. To achieve this, the substitutions

$$
\begin{aligned}
n & \rightarrow \frac{l}{\Delta l}, \\
k & \rightarrow \frac{x}{\Delta l}, \\
Z_{L} & \rightarrow \Delta l \cdot Z_{L}^{\prime}
\end{aligned}
$$

and

$$
Z_{Q} \rightarrow \frac{Z_{Q}^{\prime}}{\Delta l}
$$

are applied to Equation (7). Afterward, the limit as $\Delta l \rightarrow 0$ is calculated. In this way, the complex amplification factor (7) turns into the transfer function 


$$
H=\frac{\cosh \left((l-x) \sqrt{\frac{Z_{L}^{\prime}}{Z_{Q}^{\prime}}}\right)+\frac{\sqrt{Z_{L}^{\prime} Z_{Q}^{\prime}}}{Z_{T}} \sinh \left((l-x) \sqrt{\frac{Z_{L}^{\prime}}{Z_{Q}^{\prime}}}\right)}{\cosh \left(l \sqrt{\frac{Z_{L}^{\prime}}{Z_{Q}^{\prime}}}\right)+\frac{\sqrt{Z_{L}^{\prime} Z_{Q}^{\prime}}}{Z_{T}} \sinh \left(l \sqrt{\frac{Z_{L}^{\prime}}{Z_{Q}^{\prime}}}\right)} .
$$

With this, one can now calculate the voltage

$$
u(x, t)=H \cdot U_{0} \mathrm{e}^{j \omega t}
$$

at time $t$ at any point $x$ in the range $0, \cdots, l$ for the input voltage

$$
u_{0}=u(x=0, t)=U_{0} \mathrm{e}^{j \omega t},
$$

which is imposed by the voltage source.

The parameters $Z_{L}^{\prime}$ and $Z_{Q}^{\prime}$ are impedances related to a unit of length. The parameter $Z_{L}^{\prime}$ is the serial impedance per unit length and has the unit $\Omega / \mathrm{m}$. Because the shunt impedance decreases with increasing length of the transmission line, $Z_{Q}^{\prime}$ has the unit $\Omega \cdot \mathrm{m}$. This fact also justifies the differences between the substitutions (13) and (14).

With the solution (16) found for the voltage, the derivation for the solution for the current flowing along the transmission line is now straightforward. As one can see, for each loop in Figure 1, the equation $u_{k-1}=Z_{L} i_{k}+u_{k}$ applies. From this, it follows that

$$
i_{k}=-\frac{U_{k}-U_{k-1}}{Z_{L}} \mathrm{e}^{j \omega t} .
$$

By inserting equation (5), one obtains

$$
i_{k}=-\frac{G(k, n)-G(k-1, n)}{Z_{L}} U_{0} \mathrm{e}^{j \omega t} .
$$

If one once more applies the substitutions (11), (12), (13) and (14) and then carries out the calculation of the limit $\Delta l \rightarrow 0$, then one obtains

$$
i(x, t)=-\frac{1}{Z_{L}^{\prime}} \frac{\partial H}{\partial x} U_{0} \mathrm{e}^{j \omega t} .
$$

A comparison of Equation (20) with Equation (16) shows that the equation

$$
i(x, t)=-\frac{1}{Z_{L}^{\prime}} \frac{\partial u(x, t)}{\partial x}
$$

applies. Rearrangement of the terms results in

$$
\frac{\partial u(x, t)}{\partial x}=-Z_{L}^{\prime} i(x, t)
$$

which is the first of the telegrapher's equations. Differentiating equation (20) with respect to $x$ gives

$$
\frac{\partial i(x, t)}{\partial x}=-\frac{1}{Z_{L}^{\prime}} \frac{\partial^{2} H}{\partial x^{2}} U_{0} \mathrm{e}^{j \omega t} .
$$

It can be verified that 


$$
\frac{\partial^{2} H}{\partial x^{2}}=\frac{Z_{L}^{\prime}}{Z_{Q}^{\prime}} H
$$

Inserting this finding into Equation (23), in combination with Equation (16), gives the second of the two telegrapher's equations:

$$
\frac{\partial i(x, t)}{\partial x}=-\frac{1}{Z_{Q}^{\prime}} u(x, t) .
$$

If one now replaces the length-related impedances in the Equations (22) and (25) with the usual definitions $Z_{L}^{\prime}=j \omega L^{\prime}+R^{\prime}$ and $1 / Z_{Q}^{\prime}=j \omega C^{\prime}+G^{\prime}$, then one obtains the telegrapher's equations in the form that is commonly used in electrical engineering ([4], p. 22):

$$
\begin{aligned}
& \frac{\partial u(x, t)}{\partial x}=-\left(R^{\prime}+L^{\prime} \frac{\partial}{\partial t}\right) i(x, t) \\
& \frac{\partial i(x, t)}{\partial x}=-\left(G^{\prime}+C^{\prime} \frac{\partial}{\partial t}\right) u(x, t)
\end{aligned}
$$

Finally, this result shows that the Equations (16) and (20) are valid solutions of the telegrapher's Equations (26) and that the Equation (15) is the general transfer function of a transmission line.

\section{Interpretation}

\subsection{Definitions}

To analyze the transfer function (15), it is necessary to decompose the function into frequency and phase response. Let

$$
H:=a \mathrm{e}^{j \phi}
$$

with the real-valued amplitude $a$ and the real-valued phase $\phi$. For the frequency response $a$, the equation

$$
a=\sqrt{H \cdot H^{*}}
$$

applies because

$$
H^{*}:=a \mathrm{e}^{-j \phi} .
$$

As a result,

$$
H=\sqrt{H \cdot H^{*}} \mathrm{e}^{j \phi},
$$

which, by rearranging, leads to

$$
\phi=-j \log \left(\frac{H}{\sqrt{H \cdot H^{*}}}\right) .
$$

With knowledge of the phase $\phi$, it is possible to calculate the phase delay $\tau_{p}$, because

$$
\tau_{p}:=-\frac{\phi}{\omega}=\frac{j}{\omega} \log \left(\frac{H}{\sqrt{H \cdot H^{*}}}\right) .
$$

However, the group delay ([6], p. 232) is defined by 


$$
\tau_{g}:=-\frac{\partial \phi}{\partial \omega}=j \frac{\sqrt{H \cdot H^{*}}}{H} \frac{\partial}{\partial \omega} \frac{H}{\sqrt{H \cdot H^{*}}} .
$$

For a transmission line of length $l$, the phase velocity is therefore

$$
v_{p}:=\frac{l}{\tau_{p}},
$$

and for the group velocity, the following applies:

$$
v_{g}:=\frac{l}{\tau_{g}} .
$$

\subsection{Interpretation Using an Example}

The transfer function (15) derived in section 0 is now interpreted using an example. The line parameters of a typical $75 \Omega$ coaxial cable are:

- insulator capacitance $C^{\prime}=50 \mathrm{pF} / \mathrm{m}$.

- loop inductance $L^{\prime}=280 \mathrm{nH} / \mathrm{m}$.

- loop resistance $R^{\prime}=120 \mathrm{~m} \Omega / \mathrm{m}$.

Furthermore, a small resistance $R_{s}^{\prime}=4 \times 10^{-4} \Omega \cdot \sqrt{\mathrm{s}} / \mathrm{m}$ is assumed, which depends on the square root of the angular frequency. Note that the dependence on the square root of the angular frequency is typical for the skin effect ([7], p. $\sim 451$ ). The insulator conductance $G^{\prime}$, which usually occurs in the telegrapher's Equations (26), is assumed to be zero and is thus neglected.

From this results

$$
\begin{aligned}
Z_{L}^{\prime} & =j \omega L^{\prime}+R^{\prime}+\sqrt{|\omega|} R_{s}^{\prime} \\
& =0.12 \Omega / \mathrm{m}+\sqrt{|\omega|} 4 \times 10^{-4} \Omega \cdot \sqrt{\mathrm{s}} / \mathrm{m}+j \omega 2.8 \times 10^{-7} \Omega \cdot \mathrm{s} / \mathrm{m}
\end{aligned}
$$

and

$$
Z_{Q}^{\prime}=\frac{1}{j \omega C^{\prime}}=-j \frac{1}{\omega} 2 \times 10^{10} \Omega \cdot \mathrm{m} / \mathrm{s} .
$$

Figure 2 shows the frequency response (28) for an unterminated transmission line, i.e., for $Z_{T} \rightarrow \infty$, for the corresponding parameters at cable lengths of $l=100,200,300$ and $1000 \mathrm{~m}$ as a function of the frequency $f=\omega /(2 \pi)$.

Figure 3 shows the corresponding phase velocities (34) in the low-frequency range in relation to the speed of light in a vacuum, $c$. It is remarkable that the phase velocities are clearly higher than $c$, although, as must be emphasized, no amplification or attenuation occurs in this frequency range (see Figure 2). Only the 1000-m-long cable shows the behavior that one would expect.

At this point, one might suspect that the previously performed calculations contain an error. However, this is not the case, because the superluminal phase velocities shown in Figure 3 actually occur in this form, as shown in a recent experimental work [8]. In fact, a comparison between Figure 3 and Figure 4 in [8] shows a remarkably high agreement.

The effect of the superluminal phase velocities in the low-frequency range disappears almost completely when the coaxial cable is terminated with a $75 \Omega$ 


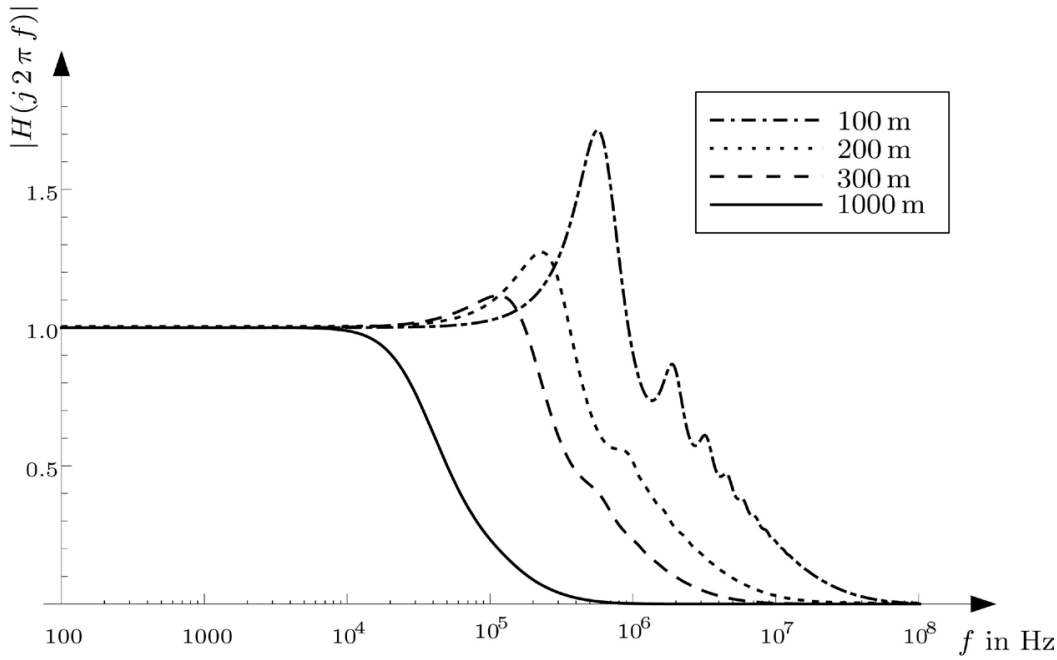

Figure 2. Frequency response (no termination, no measures against the skin effect).

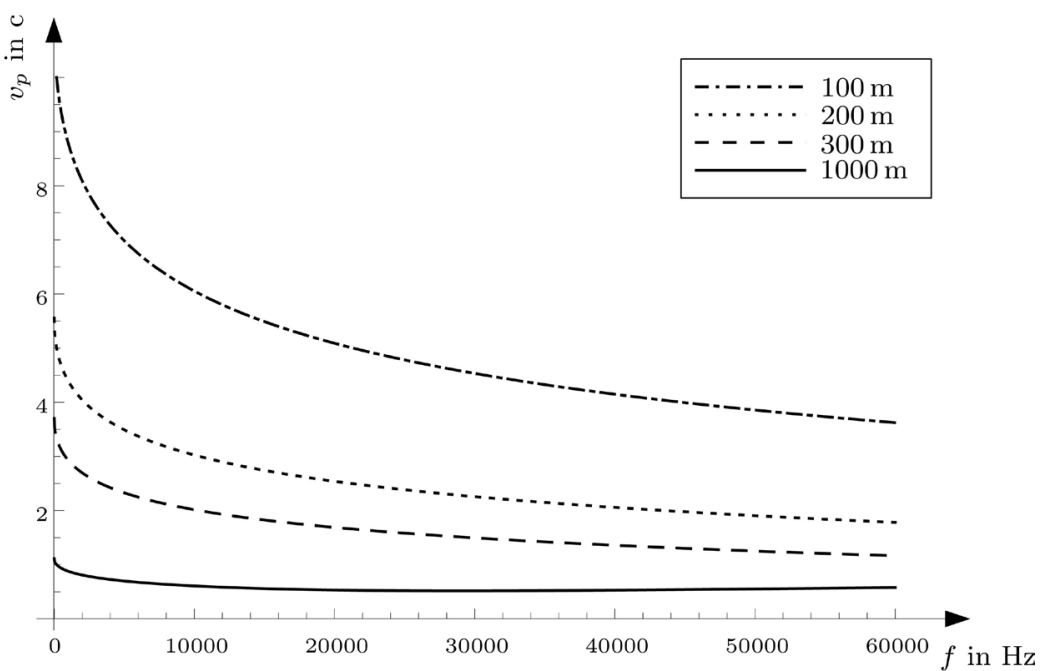

Figure 3. Phase velocities in the low-frequency range (no termination, no measures against the skin effect).

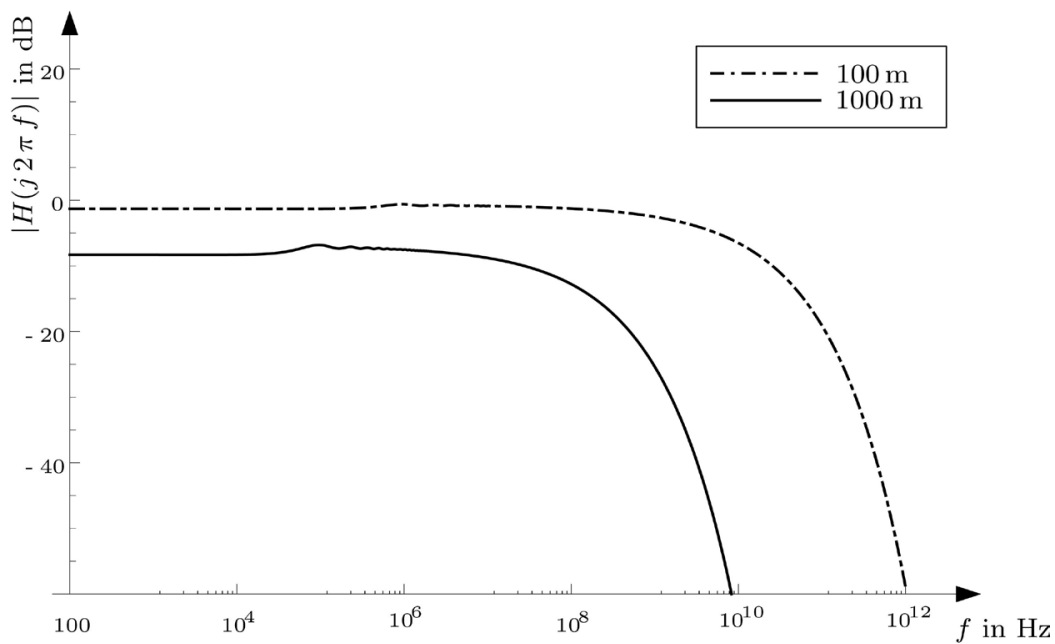

Figure 4. Frequency response in $\mathrm{dB}$ (termination with $75 \Omega$, high-frequency cable). 
resistor. This is a common practice in applied electrical engineering and is carried out with the aim of minimizing reflections.

That reflections occur in the high-frequency range can be seen based on the frequency response in Figure 2, because resonances occur at specific frequencies. This effect is even more pronounced for cables that are optimized for high-frequency applications. Figure 5 shows such a frequency response for an unterminated transmission line. In comparison to Figure 2, only the parameter of the skin effect is different, because in Figure 5, this parameter has the value $R_{s}^{\prime}=4 \times 10^{-6} \Omega \cdot \sqrt{\mathrm{s}} / \mathrm{m}$.

When using a terminating resistor of $75 \Omega$ the resonances disappear almost completely, as shown in Figure 4. This finding means that the common practice of terminating coaxial cables with a resistor is useful and necessary, if the frequency range of the signal to be transmitted is in the high-frequency range.

However, in the low-frequency range, it may be advantageous not to terminate the cable, to prevent unnecessary current flow. The corresponding cut-off frequency for the need to use a terminating resistor depends mainly on the length of the line and can be determined, as demonstrated, by means of the transfer function calculated in section 2 . Another reason for deliberately omitting the terminating resistor is to achieve the highest possible phase and group velocities in the low-frequency range, because this effect can be exploited technologically, as the following sections show.

Figure 6 shows the group velocities calculated with the formulas (33) and (35) for the same parameters as those in Figure 3. As can be seen, the group velocities in the low-frequency range are also far greater than the speed of light in a vacuum, as long as the cable is not too long and no termination is carried out.

However, by attaching a terminating resistor, the effect disappears almost completely, as shown in Figure 7.

It is further pointed out that the phase velocities are almost identical to the

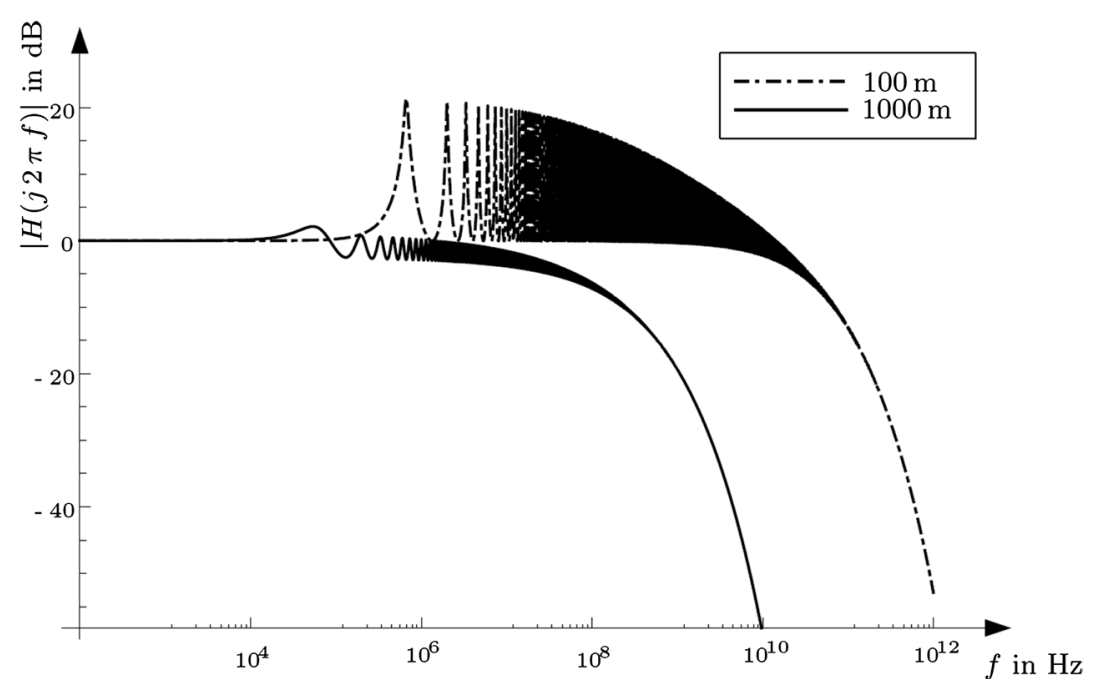

Figure 5. Frequency response in $\mathrm{dB}$ (no termination, high-frequency cable). 


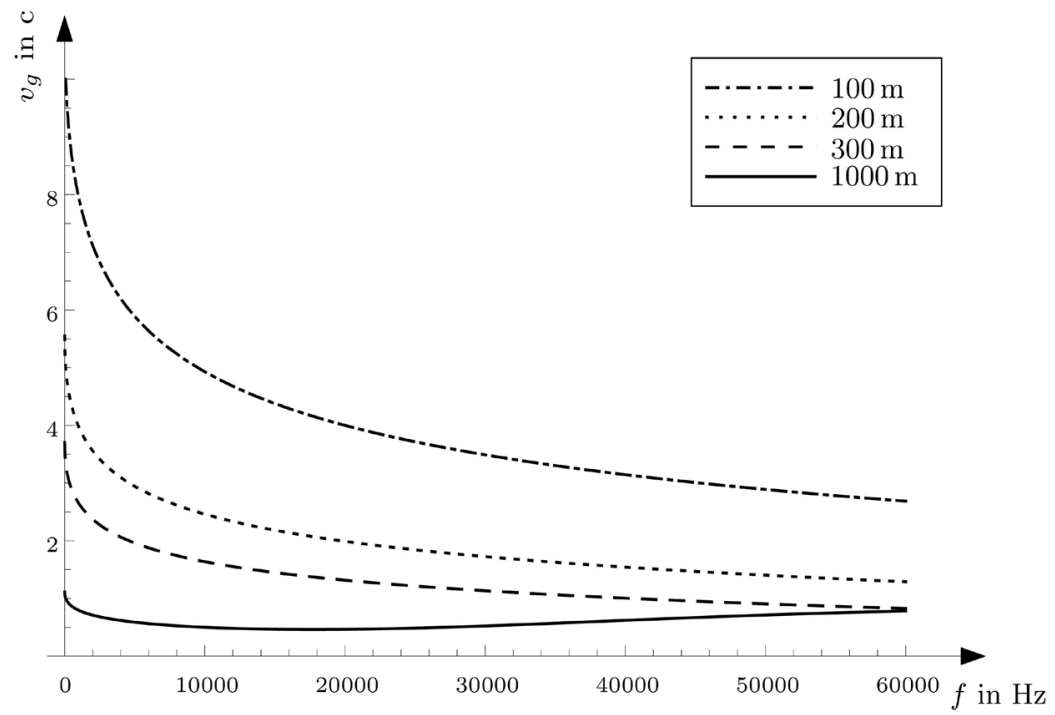

Figure 6. The group velocities compared to the phase velocities in Figure 3 (no termination).

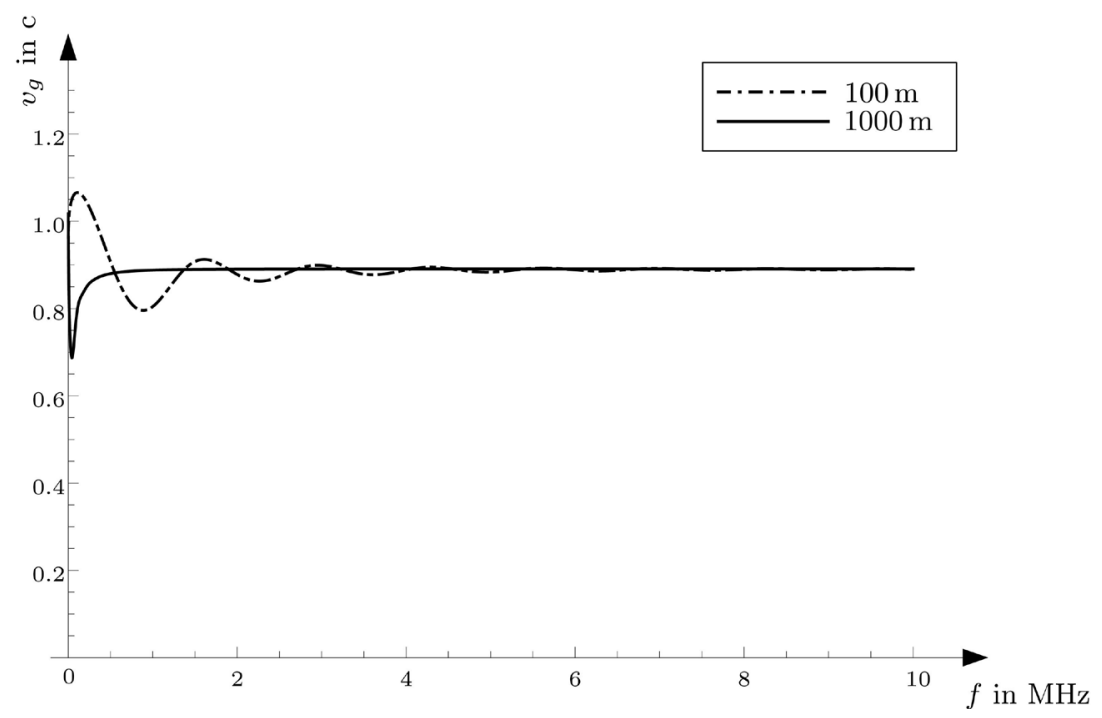

Figure 7. The group velocities when using a terminating resistor of $75 \Omega$. The parameters are identical to those in Figure 6.

group velocities, as can be seen by comparing Figure 3 and Figure 6.

\subsection{Signal Velocity}

It has already been shown that when using a terminating resistor, the phase and group velocities become so small that they are less than the speed of light in a vacuum. The generally accepted rule that information cannot be propagated at a superluminal velocity is thus correct for terminated transmission lines (see Figure 7).

However, if the termination is omitted, i.e., for $Z_{T} \rightarrow \infty$, a coaxial cable of, e.g., $100 \mathrm{~m}$ in length with the standard line constants mentioned in section 3.2 behaves very oddly, because a low-frequency voltage signal applied to the input 
appears unchanged at the output, almost without any time delay. To show this, in the first step, the termination is removed in the transfer function (15), i.e., the limit $Z_{T} \rightarrow \infty$ is calculated for $x=l$. This gives

$$
H_{o}:=\left.\lim _{Z_{T} \rightarrow \infty} H\right|_{x=l}=\cosh \left(l \sqrt{\frac{Z_{L}^{\prime}}{Z_{Q}^{\prime}}}\right)^{-1} .
$$

Now, it can be seen that the terms with the characteristic impedance $\sqrt{Z_{L}^{\prime} Z_{Q}^{\prime}}$ have disappeared. By inserting the line constants (36) and (37) and neglecting the skin effect

$$
H_{o}=\cosh \left(l \sqrt{j \omega C^{\prime} R^{\prime}-C^{\prime} L^{\prime} \omega^{2}}\right)^{-1}
$$

follows. For sufficiently small $\omega$, it is possible to develop $H_{o}$ into a Taylor series and to omit the third-order and higher terms. As a result, the approximation

$$
H_{o} \approx 1-\frac{1}{2} j C^{\prime} l^{2} R^{\prime} \omega+\left(\frac{1}{2} C^{\prime} L^{\prime} l^{2}-\frac{5}{24} C^{\prime 2} R^{\prime 2} l^{4}\right) \omega^{2}
$$

is obtained.

By applying equations (28) and (31) and neglecting all terms of third and higher order with respect to $\omega$, one obtains the frequency response

$$
a \approx 1+\frac{1}{12}\left(6 L^{\prime} C^{\prime} l^{2}-\left(R^{\prime} C^{\prime} l^{2}\right)^{2}\right) \omega^{2}
$$

and the phase response

$$
\phi \approx-\frac{1}{2} R^{\prime} C^{\prime} l^{2} \omega .
$$

Therefore, the complete transfer function has the form

$$
H_{o} \approx\left(1+\kappa \omega^{2}\right) \mathrm{e}^{-j \omega \tau}
$$

with the frequency independent constants

$$
\tau:=\frac{1}{2} R^{\prime} C^{\prime} l^{2}
$$

and

$$
\kappa:=\frac{1}{2} L^{\prime} C^{\prime} l^{2}-\frac{1}{3} \tau^{2} .
$$

By inserting the transfer function (43) into the formula of the phase delay (32) follows after a short calculation

$$
\tau_{p}=\tau .
$$

Inserting of the transfer function (43) into the formula of the group delay (33) results in

$$
\tau_{g}=\tau .
$$

It is obvious that phase delay and group delay are identical and independent of the frequency. This means that there is no dispersion at all and that the signal 
shape is preserved.

Now, let $S_{i n}(\omega)$ be the Fourier transform of a time signal $s_{i n}(t)$, i.e., it shall apply that

$$
S_{i n}(\omega):=\int_{-\infty}^{+\infty} s_{i n}(t) \mathrm{e}^{-j \omega t} \mathrm{~d} t
$$

If the signal $s_{i n}(t)$ is given to the input of a transmission line with the transfer function (43), then the equation

$$
S_{\text {out }}(\omega)=\left(1+\kappa \omega^{2}\right) \mathrm{e}^{-j \omega \tau} \cdot S_{\text {in }}(\omega)
$$

applies to the Fourier transform $S_{\text {out }}(\omega)$ of the output signal $s_{\text {out }}(t)$. With the inverse Fourier transform, one obtains

$$
\begin{aligned}
S_{\text {out }}(t) & =\frac{1}{2 \pi} \int_{-\infty}^{+\infty}\left(1+\kappa \omega^{2}\right) S_{\text {in }}(\omega) \mathrm{e}^{j \omega(t-\tau)} \mathrm{d} \omega \\
& =s_{\text {in }}(t-\tau)+\frac{\kappa}{2 \pi} \int_{-\infty}^{+\infty} \omega^{2} S_{\text {in }}(\omega) \mathrm{e}^{j \omega(t-\tau)} \mathrm{d} \omega \\
& =s_{\text {in }}(t-\tau)-\frac{\kappa}{2 \pi} \frac{\mathrm{d}^{2}}{\mathrm{~d} t^{2}} \int_{-\infty}^{+\infty} S_{i n}(\omega) \mathrm{e}^{j \omega(t-\tau)} \mathrm{d} \omega,
\end{aligned}
$$

i.e., the output signal is

$$
s_{\text {out }}(t)=s_{\text {in }}(t-\tau)-\kappa s_{\text {in }}^{\prime \prime}(t-\tau) .
$$

Using the example parameters of section 3.2, for a cable with a length of 100 $\mathrm{m}$, one obtains the time constant

$$
\tau=30 \mathrm{~ns}
$$

and the attenuation

$$
\kappa \approx 7 \times 10^{-14} \mathrm{~s}^{-2} .
$$

Thus, the effect of the 100 -m-long cable is mainly to delay the input signal by 30 ns. However, this finding means that the signal propagates effectively at a speed of more than 11 times the speed of light in a vacuum in the $100-\mathrm{m}$-long cable. Furthermore, because the signal does not change as it propagates through the cable, this velocity is the signal velocity and not simply a phase or group velocity.

By looking at Equation (44), it can be seen that the time constant $\tau$, and thus the time delay, becomes zero when the resistance $R^{\prime}$ disappears. Therefore, it seems obvious to assume that signals in superconductors propagate at infinitely high signal velocities. However, in reality, this might not be the case, because superconductors have a resistance for alternating currents [9] and because the skin effect also occurs in these instances, which was neglected in section $3.3^{1}$.

To include the skin effect in Equation (40), the frequency-independent resistance $R^{\prime}$ must be replaced by the frequency-dependent resistance $R^{\prime}+\sqrt{|\omega|} R_{s^{\prime}}$. From this, $\tau$ in Equation (44) obtains the additional term

${ }^{1}$ However, the author does not know of any experiments in which the signal velocity was measured at low frequencies in electrically short superconductors. 


$$
\tau_{d}(\omega):=\frac{1}{2} \sqrt{|\omega|} R_{s}^{\prime} C^{\prime} l^{2}
$$

and one must replace $\tau$ in Equations (43) and (45) accordingly with $\tau+\tau_{d}(\omega)$.

If one ignores the very small attenuation $\kappa$, then for the Fourier transform $S_{\text {out }}(\omega)$, the equation

$$
S_{\text {out }}(\omega)=\mathrm{e}^{-j \omega \tau} \cdot \mathrm{e}^{-j \omega \tau_{d}(\omega)} \cdot S_{\text {in }}(\omega)
$$

applies. The first factor causes the already-explained time delay with the time constant $\tau$. The second factor also produces a time delay, but this time delay is now frequency-dependent and grows with increasing frequency. This finding means that components with lower frequencies transmit faster than components with higher frequencies, which is known as normal dispersion. For appropriately narrow-band signals, however, the effect can be neglected and one obtains in the time domain the frequency independent approximation

$$
s_{\text {out }}(t) \approx S_{\text {in }}\left(t-\left(\tau+\tau_{d}\left(\omega_{c}\right)\right)\right),
$$

with $\omega_{c}$ being the carrier frequency of the signal.

\subsection{Superluminal Signal Transmission over Long Distances}

Until now, it has only been shown that signals move at superluminal velocities if the signal frequencies are sufficiently low and the transmission lines are sufficiently short. One might be inclined to dismiss this phenomenon as an interesting but practically irrelevant effect, because low-frequency signals are broad and elongated and are therefore not suitable for transmitting messages over short distances. However, this assumption would be a fallacy, because in principle, it is possible to convey this effect to cables of almost any length.

The basic idea is to connect two electrically short cables together in such a way that no current can flow from the first into the second. This can be realized, for example, as suggested in [8], by means of a unity-gain buffer amplifier. An ideal unity-gain buffer amplifier is a circuit that has an infinitely high input resistance and outputs the voltage present at the input without any delay and with an infinitely low output impedance. Figure 8 shows the equivalent circuit diagram of an ideal unity-gain buffer amplifier.

If one connects an electrically short cable with an ideal unity-gain buffer amplifier, then $Z_{T}$ in Equation (15) approaches infinity and the transfer function of the cable corresponds to Equation (38) and thus, for sufficiently low frequencies, to approximation (43) as well. However, the transfer function of an ideal unity-gain buffer amplifier is equal to one. Consequently, the total transfer function of the cable together with the buffer amplifier is also given by Equation (43).

The output of the buffer amplifier can now be connected once more with another segment of electrically short cable and another ideal unity-gain buffer amplifier. It is obvious that further segments can be connected accordingly and that there is no limit to the total number of segments, at least within the context of the model assumptions. The total transfer function $H_{T}$ for $n$ segments can 


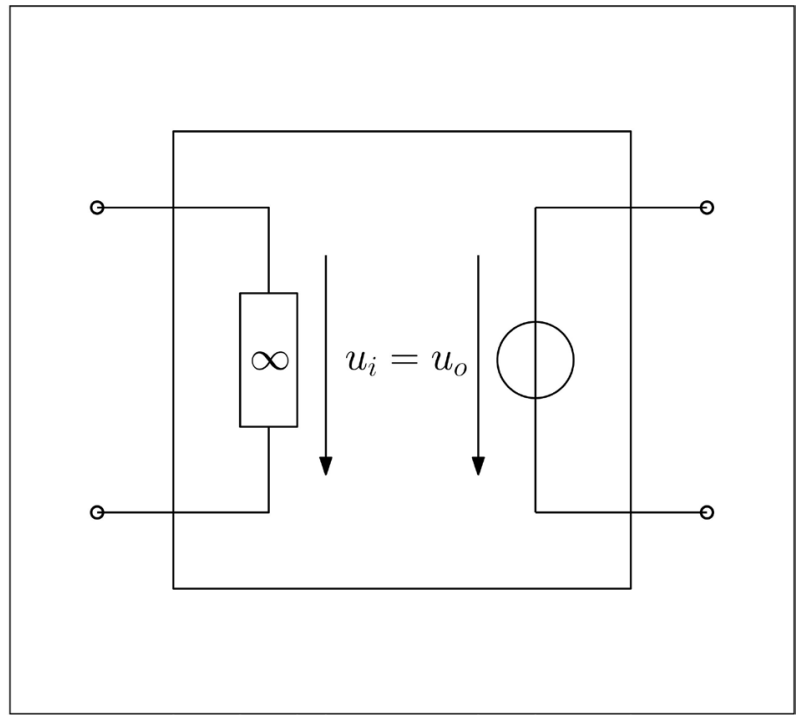

Figure 8. Ideal unity-gain buffer amplifier.

be obtained by multiplying the transfer functions of all individual segments according to the two-port network theory, i.e.,

$$
H_{T}=H_{o}^{n} \approx\left(1+\kappa \omega^{2}\right)^{n} \mathrm{e}^{-j n \omega \tau} \approx\left(1+n \kappa \omega^{2}\right) \mathrm{e}^{-j n \omega \tau} .
$$

The output signal $s_{\text {out }}(t)$ to a given input signal $s_{i}(t)$, which is given to the input of the entire transmission line of length $n \cdot l$, is therefore

$$
s_{\text {out }}(t)=s_{\text {in }}(t-n \tau)-n \kappa s_{\text {in }}^{\prime \prime}(t-n \tau) .
$$

The fact that an ideal unity-gain buffer amplifier does not exist, does not diminish this result. Instead of ideal buffer amplifiers, real operational amplifiers can be used, which in the low-frequency range are also very close to ideal delay elements and which have time constants as low as $\tau_{B A}=25 \mathrm{~ns}$ [8]. With this, the output signal is

$$
s_{\text {out }}(t)=s_{\text {in }}\left(t-n\left(\tau+\tau_{B A}\right)\right)-n \kappa s_{\text {in }}^{\prime \prime}\left(t-n\left(\tau+\tau_{B A}\right)\right),
$$

which with $\tau=30 \mathrm{~ns}$ and $l=100 \mathrm{~m}$ still corresponds to a signal velocity of more than six times the speed of light in a vacuum.

It is pointed out that for large $n$, the influence of the second term becomes more important because of $n \kappa$. However, even this term does not lead to a dispersion of the signal. For example, if a Gaussian pulse

$$
s(t)=\mathrm{e}^{-2 \pi^{2} f_{B}^{2} t^{2}} \cos \left(2 \pi f_{c} t\right)
$$

modulated with the carrier frequency $f_{c}$ is used as a signal, then the second derivative with respect to $t$ still has the same envelope, because the expression is given by

$$
s^{\prime \prime}(t)=\mathrm{e}^{-2 \pi^{2} f_{B}^{2} t^{2}}\left(k_{1} t^{2} \cos \left(2 \pi f_{c} t\right)+k_{2} t \sin \left(2 \pi f_{c} t\right)\right)
$$

with the constants $k_{1}$ and $k_{2}$, which are not of interest here.

If one inserts the signal (60) and its second derivative (61) into Equation (59) 
and calculates the envelope $\mathcal{E}$ on both sides, then one obtains

$$
\mathcal{E}\left\{s_{\text {out }}(t)\right\}=\mathcal{E}\left\{s_{\text {in }}\left(t-n\left(\tau+\tau_{B A}\right)\right)\right\} .
$$

It should be noted that from the point of view of electrical engineering, the envelope is also a signal, which in this case travels at a signal velocity of

$$
v_{s}=\frac{n l}{n\left(\tau+\tau_{B A}\right)}=\frac{l}{\tau+\tau_{B A}} .
$$

Assuming that $n=3 \times 10^{6}, f_{b}=5000 \mathrm{~Hz}$ and $f_{c}=30000 \mathrm{~Hz}$, then it follows, based on the already-used parameters $l=100 \mathrm{~m}, \tau_{B A}=25 \mathrm{~ns}$ and $\tau=30 \mathrm{~ns}$, that the pulse requires $3 \times 10^{6} \times 55 \mathrm{~ns}=0.165 \mathrm{~s}$ to pass the entire transmission line, although the pulse itself has only a temporal width of $1 / f_{b}=200 \mu \mathrm{s}$. A light pulse in a vacuum would require one second for the same distance.

\section{Conclusions}

This article showed mathematically that according to the telegrapher's equations, it should be possible to transmit messages at signal speeds that significantly exceed the speed of light in a vacuum. The fact that this has not yet been explicitly reported is probably due to several causes, such as the strong focus on long cable lengths and high frequencies. Furthermore, it can certainly be assumed that the majority of today's scientists consider the telegrapher's equations to be completely understood. This set of circumstances is the only reasonable justification for why solutions with limited validity and resulting conclusions from old articles or textbooks have been accepted for so long without being questioned.

It is pointed out that this article would not have been written if experimental facts had not provided evidence that at low frequencies and short cable lengths, the expectations do not correspond to the measured results [8]. This article shows that the high phase velocities measured in [8] although not consistent with the special theory of relativity, are fully compatible with the transmission line theory. Furthermore, the idea is confirmed that buffer amplifier chains should in principle allow the transmission of signals over long distances at signal speeds far greater than the speed of light in a vacuum.

To demonstrate this principle, this article starts by solving the telegrapher's equations in an unusual but effective way. The approach used in the literature is to choose a parametric model and then to deduce the missing parameters from boundary conditions and constraints. However, in this article, the solution is derived directly using circuit theory. This approach ensures that all boundary conditions are correctly considered. The resulting solution of the telegrapher's equations is very general and applies to both electrically long and electrically short transmission lines, which in addition may be either open or terminated. Furthermore, the solution also provides the transfer function of the transmission line, which allows an interpretation using the methods of signal processing. 
After deducing the solution, the article analyses the transfer function of the transmission line. It is concluded that transmission lines of length $I$ behave like ideal delay elements, if:

1) The transmission lines are not terminated, i.e., current flow and energy transport are minimized, and if

2) The transmitted signals contain only frequencies for which the frequency response is close to one.

Furthermore, a formula for the signal velocity

$$
v_{s}=\frac{2}{R^{\prime} C^{\prime} l}
$$

is provided, which is valid under the stated conditions.

The article concludes with an analysis of the idea proposed in [8] to make the effect available for long-distance communication by connecting many short cables in a feedback-free way. In this case, the transfer functions of all segments multiply and it can be mathematically shown that the total transfer function has the same signal velocity as a single segment alone.

The conclusion of this article is that the fundamentals of electrical engineering, which have worked perfectly well for more than a hundred years, allow signals to be transmitted over any distance at signal speeds that are significantly higher than the speed of light in a vacuum. The fact that this contradicts the special theory of relativity is irrelevant for electrical engineering and a question that must be answered by physics.

\section{Acknowledgements}

I thank the editors and reviewers for their support. I also thank Farhad Vedad for repeating the experimental study. Finally, I would like to thank my wife for her patience and support.

\section{Conflicts of Interest}

The author declares no conflicts of interest regarding the publication of this paper.

\section{References}

[1] Nimtz, G. and Enders, A. (1992) On Superluminal Barrier Traversal. Journal de Physique I, 2, 1693-1698. https://doi.org/10.1051/jp1:1992236

[2] Mitchell, M.W. and Chiao, R.Y. (1998) Causality and Negative Group Delays in a Simple Bandpass Amplifier. American Journal of Physics, 66, 14 https://doi.org/10.1119/1.18813

[3] Wang, L., Kuzmich, A. and Dogariu, A. (2000) Gain-Assisted Superluminal Light Propagation. Nature, 406, 277-279. https://doi.org/10.1038/35018520

[4] Moore, R.K. (1960) Traveling-Wave Engineering. McGraw-Hill, New York.

[5] Magnusson, P.C., Weisshaar, A., Tripathi, V.K. and Alexander, G.C. (2017) Transmission Lines and Wave Propagation. CRC Press, Boca Ration, London, New York, Washington D.C. 
[6] Collin, R.E. (1991) Field Theory of Guided Waves. IEEE Press, New York. https://doi.org/10.1109/9780470544648

[7] Kraus, J.D. (1984) Electromagnetics. McGraw-Hill, New York.

[8] Kühn, S. (2019) Electronic Data Transmission at Three Times the Speed of Light and Data Rates of 2000 Bits Per Second in Buffer Amplifier Chains. Preprint.

[9] von Laue, M. (1952) Theory of Superconductivity. Academic Press, New York. 